\title{
Fueter Mapping Theorem in Hypercomplex Analysis
}

\author{
January 15, 2014
}

Tao Qian

University of Macau, fsttq@umac.mo

\begin{abstract}
Fueter's Theorem offers a method that conveys certain holomorphic functions in one complex variable to quaternionic regular (monogenic) functions. Ever since the theorem was proved in 1935, it underwent several main generalizations. Those, not only are based on its own interest, but also motivated by applications found in other areas of mathematics, such as functional calculus of operators. This article serves as a survey on Fueter's Theorem, its generalizations and applications.
\end{abstract}

Key words Quaternion, Fueter's Theorem, Clifford algebra, Sce's Theorem, fractional powers of Laplacian, $H^{\infty}$ Functional Calculus

AMS subject classifications. 62D05, 30D10, 42B35

\section{Introduction}

Fueter's Theorem and its generalizations, on one hand, are quite artificial: One uses a uniform format to construct new monogenic and new harmonic functions, but one can only mathematically prove each particular case, one does not really understand why one can do so in general. On the other hand, this is an extremely interesting subject showing beauty and application of mathematics. Mathematical beauty is its ideality: what one could expect would eventually be proved true, and is something you admire and enjoy, and you can even use. Fueter's Theorem and its generalizations (called as the Fueter type theorems) possess all these aspects. In $\S 2$ Fueter's Theorem is presented. In $\S 3$ M. Sce's and T. Qian's generalizations of Fueter's Theorem to Euclidean spaces $\mathbf{R}_{1}^{n}$ are introduced. Sce's generalization is based on integer powers of the Laplacian in odd dimensions $n$. Qian's generalization uses the Fourier multiplier definition of fractional powers of the Laplacian in any dimension $n$. Consistency of the two different approaches in their common parts is recalled. $\S 4$ presents the generalizations initiated by F. Sommen, called as Fueter's Theorem with a harmonic factor. After Sommen's initialization for $n$ being odd, Kou and Qian joined in, and work out together with Sommen the cases for $n$ being even. Qian and Sommen subsequently proposed an analogous mechanism to derive harmonic 
functions in higher dimensions. With D. Penã Penã's joining to work the subjects for multidimensions are further developed that cover the Fueter type Theorems of poly-axis types and with harmonic factors. $\S 5$ summarizes applications of Fueter's Theorem and its generalizations. Apart from generating kernels and Clifford-Hermite and Clifford Gegenbauer Polynomials we provide in detail the applications in constructing $H^{\infty}$-functional calculi of Dirac operators on Lipschitz perturbations of the higher dimensional spheres. This essay ends in $\S 7$ with some information on inverse Fueter's Theorem that has been pursuing mainly by F. Colombo and I. Sabadini et al. Although it is still on going, a significant part has been done with applications on functional calculus of non-commutative operators.

The author wishes to sincerely thank I. Sabadini, F. Colombo and D. Peña Peña for helpful information and remarks on the subject which greatly contribute to the writing out of this essay.

\section{Fueter's Theorem}

Fueter's Theorem concerns the real quaternionic space

$$
\mathcal{H}=\left\{q=q_{0}+q_{1} \mathbf{i}+q_{2} \mathbf{j}+q_{3} \mathbf{k} \mid q_{l} \in \mathbf{R}, l=0,1,2,3\right\}
$$

where $\mathbf{i}, \mathbf{j}, \mathbf{k}$ are the usual quaternionic imaginary elements satisfying

$$
\mathrm{ij}=\mathrm{k}, \mathbf{j k}=\mathbf{i}, \mathbf{k i}=\mathbf{j}, \mathbf{i j}=-\mathbf{j i}, \mathbf{j k}=-\mathbf{k j}, \mathbf{k i}=-\mathbf{i k},
$$

and

$$
\mathbf{i}^{2}=\mathbf{j}^{2}=\mathbf{k}^{2}=-1 .
$$

We usually write $q=q_{0}+\underline{q}, \underline{q}=q_{1} \mathbf{i}+q_{2} \mathbf{j}+q_{3} \mathbf{k}$. We will use the quaternionic conjugation defined by $\bar{q}=q_{0}-\underline{q}$. $\mathcal{H}$ forms a non-commutative real algebra and a four-dimensional linear algebra. Easy computation shows that it is divisable: For $0 \neq q \in \mathcal{H}$ there holds

$$
q^{-1} q=q q^{-1}=1
$$

where

$$
q^{-1}=\frac{\bar{q}}{|q|^{2}}, \quad|q|^{2}=q_{0}^{2}+q_{1}^{2}+q_{2}^{2}+q_{3}^{2}
$$

Associated with the quaternionic space there is quaternionic analysis which is similar to complex analysis. In particular, in $\mathcal{H}$ there is a Cauchy's theory: There are analogous subjects, including the Cauchy kernel, the Cauchy Theorem, and the Cauchy integral. In quaternionic analysis the analogy of complex holomorphic functions are quaternionic regular functions, that is defined through the quaternionic Cauchy-Riemann operator (also called Dirac differential operator)

$$
D=\partial_{0}+\mathbf{i} \partial_{1}+\mathbf{j} \partial_{2}+\mathbf{k} \partial_{3} .
$$

Precisely, if $f$ is a quaternionic-valued function in a quaterninic variable and $D f=0$ in a domain of the space, then $f$ is said to be left-regular in the domain, and if $f D=0$, right-regular in the domain. For a detailed exposition of quaternionic analysis the reader is referred to A. Sudbery [Su] or R. Delanghe et al [DSS]. 
N. Frobenius proved that besides the real and the complex number systems the quaternionic system is the only other linear algebra of finite dimension that at the same time is a divisable algebra. It is observed that there exists a remarkable relation between holomorphic functions in the complex system and regular functions in the quaternionic system. The formulation of this relation is the so called Fueter's Theorem.

Theorem 1 (Fueter's Theorem, 1935, [15]) Let $f_{0}=u+i v$ be a complex holomorphic function defined in a domain (i.e., an open and connected subset) $\Omega$ in the upper-half plane. Denote by $\tilde{f}_{0}$ the so-called induced function, defined in the subset

$$
\tilde{\Omega}=\left\{q=q_{0}+\underline{q} \mid\left(q_{0},|\underline{q}|\right) \in \Omega\right\}
$$

of $\mathcal{H}$ by

$$
\tilde{f}_{0}(q)=u\left(q_{0},|\underline{q}|\right)+\frac{\underline{q}}{|\underline{q}|} v\left(q_{0},|\underline{q}|\right) .
$$

Then $\Delta \tilde{f}_{0}$ is both left- and right-quaternionic regular in the relevant domain $\tilde{\Omega}$, i.e.,

$$
D \Delta \tilde{f}_{0}(q)=\Delta \tilde{f}_{0}(q) D=0, \quad q \in \tilde{\Omega},
$$

where $\Delta$ is the Laplacian for the four real variables $q_{l}, l=0,1,2,3$.

When the closure of $\Omega$ contains a non-empty open interval $(a, b)$ on the real-axis, then the closure of the induced set $\tilde{\Omega}$ contains the same interval $(a, b)$ on the $q_{0}$-axis. In the case the function $f_{0}$ should be assumed to be holomorphically extendable to the symmetric, or, in other words, intrinsic, domain $\Omega \cup \Omega^{*} \cup\left(\bar{\Omega} \cap \overline{\Omega^{*}}\right)^{o}$, where

$$
\Omega^{*}=\{z \in \mathbf{C} \mid \bar{z} \in \Omega\}
$$

the over bar ${ }^{-}$denotes the taking-closure operation applied to sets in $\mathbf{C}$, and the over circle ${ }^{o}$ denotes the taking-interior operation.

The concepts of intrinsic sets and intrinsic functions naturally fit into this theory. In the complex plane $\mathbf{C}$ a set is said to be intrinsic if it is open and symmetric with respect to the real axis; and a function $f_{0}$ is said to be an intrinsic function if it is defined in an intrinsic set satisfying $\overline{f_{0}(z)}=f_{0}(\bar{z})$ in its domain (see [44]). By writing $f_{0}(z)=u(x, y)+\mathbf{i} v(x, y)$, where $u$ and $v$ are real-valued, $z=x+i y$, the property of $f_{0}$ involving complex conjugation is equivalent to the fact that $u$ is even and $v$ is odd in the second argument. In the case, in particular, $f_{0}$ is real-valued on the real line. For more information concerning intrinsic functions in relation to Fueter's Theorem and its generalizations the reader is referred to [36] and [38].

As example, by taking $f_{0}(z)=1 / z$, the induced function for a quaternionic variable is

$$
\tilde{f}_{0}(q)=\frac{q_{0}}{q_{0}^{2}+|\underline{q}|^{2}}+\frac{\underline{q}}{|\underline{q}|} \frac{-|\underline{q}|}{q_{0}^{2}+|\underline{q}|^{2}} .
$$

Applying the Laplacian in the four real variables $q_{0}, q_{1}, q_{2}, q_{3}$ to both sides of the above relation, we obtain

$$
\Delta \tilde{f}_{0}(q)=-4 \frac{\bar{q}}{|q|^{4}}
$$


which is a constant multiple of the Cauchy kernel

$$
\frac{1}{2 \pi^{2}} \frac{\bar{q}}{|q|^{4}}
$$

in the quaternionic space ([51]). From this observation, it is, in fact, sufficient, to conclude Fueter's Theorem. The only other example that appeared in the literature before the applications given in [37] is $f_{0}(z)=\log z([51])$.

\section{Direct Generalizations of Fueter's Theorem to $\mathbf{R}^{n}$}

We assume that the reader is familiar with the standard knowledge of Clifford algebra and analysis. We use the notation $\mathbf{R}^{(n)}\left(\mathbf{C}^{(n)}\right)$ for the real (complex) Clifford algebra generated by $\mathbf{e}_{1}, \ldots, \mathbf{e}_{\mathbf{n}}$, with the properties $\mathbf{e}_{i} \mathbf{e}_{j}+\mathbf{e}_{j} \mathbf{e}_{i}=2 \delta_{i j}, i, j=1,2 \ldots, n$. Let $f_{0}$ be an intrinsic function. We deal with only holomorphic intrinsic functions $f_{0}$. Introduce the function

$$
\tilde{f}_{0}(x)=u\left(x_{0},|\underline{x}|\right)+\frac{\underline{x}}{|\underline{x}|} v\left(x_{0},|\underline{x}|\right),
$$

called the induced function of $f_{0}$ in $\mathbf{R}_{1}^{n}=\left\{x_{0}+\underline{x} \mid x_{0} \in \mathbf{R}, \underline{x}=x_{1} \mathbf{e}_{1}+\cdots+x_{n} \mathbf{e}_{n} \in \mathbf{R}^{n}\right\}$ which is defined in the induced intrinsic domain $\tilde{\Omega}$ in $\mathbf{R}_{1}^{n}$ :

$$
\tilde{\Omega}=\left\{x=x_{0}+\underline{x} \mid\left(x_{0},|\underline{x}|\right) \in \Omega\right\} .
$$

Denote by $\tau_{n}$ the Fueter mapping

$$
\tau_{n}\left(f_{0}\right)=\kappa_{n}^{-1} \Delta^{\frac{n-1}{2}} \tilde{f}_{0}, \quad \kappa_{n}=(2 \mathbf{i})^{n-1} \Gamma^{2}\left(\frac{n-1}{2}\right) .
$$

When $n$ is odd, the introduced mapping $\tau_{n}$ is a point-wise differential operator. When $n$ is even we require that the intrinsic function $f_{0}$ is a meromorphic function in the whole space $\mathbf{R}_{1}^{n}$, and $\tau_{n}$ is defined by the Fourier multiplier operator

$$
\left.\tau_{n}\left(f_{0}\right)=\mathcal{R}\left[(2 \pi \mathbf{i}|\cdot|)^{n-1}\right) \mathcal{F}\left(\tilde{f}_{0}\right)\right],
$$

where $\mathcal{F}$ and $\mathcal{R}$ are, respectively, the Fourier and inverse Fourier transformations, given, respectively, by

$$
\mathcal{F} f(\xi)=\int_{\mathbf{R}_{1}^{n}} e^{2 \pi i\langle x, \xi\rangle} f(x) d x
$$

and

$$
\mathcal{R} g(x)=\int_{\mathbf{R}_{1}^{n}} e^{-2 \pi i\langle x, \xi\rangle} g(\xi) d \xi
$$

(see [36]).

The Fourier multiplier definition for $\tau_{n}$ is applicable also for $n$ being odd. We show in [36] that the two definitions for $\tau_{n}$ for $n$ being odd are identical.

As the very first generalization of Fueter's Theorem, M. Sce published his result for $n$ being odd case in 1957, corresponding to the point-wise differentiation case. 
Theorem 2 (Sce's Theorem, 1957, [15]) If the space dimension $n$ is odd, and $f_{0}=u+i v$ is a complex holomorphic function in a domain $\Omega$ in the upper half plane, then $\tau_{n}\left(f_{0}\right)$ is both leftand right-monogenic in the relevant domain $\tilde{\Omega}$ in $\mathbf{R}_{1}^{n}$, ie.,

$$
\begin{gathered}
D\left(\tau_{n}\left(\bar{f}_{0}\right)(x)\right)=\left(\tau_{n}\left(\bar{f}_{0}\right)(x)\right) D=0, \quad x \in \tilde{\Omega}, \\
\tilde{\Omega}=\left\{x=x_{0}+\underline{x} \mid\left(x_{0},|\underline{x}|\right) \in \Omega\right\} .
\end{gathered}
$$

Next, T. Qian generalizes M. Sce's result to the cases $n$ being even in [36] by using his Fourier multiplier definition of the Fueter mapping. Let $f_{0}$ be a meromorphic intrinsic function in the whole complex domain. Expanding $f_{0}$ into its Laurent series at zero, one can see that $\tau_{n}\left(f_{0}\right)$ is reduced to a series $\sum_{k=-\infty}^{\infty} a_{k} \tau_{n}\left((\cdot)^{k}\right)$, where the coefficients $a_{k}$ are real. Thus, it suffices to only consider $f_{0}(z)=z^{k}$, where $k$ is any integer. Recall that the Kelvin inversion $I(f)(x)=E(x) f\left(x^{-1}\right)$, where $E$ is the Cauchy kernel $E(x)=\bar{x} /|x|^{n+1}$, maps the left- and right-monogenic functions to, respectively, left- and right monogenic function. We define, for any positive integer $k$,

$$
P^{(-k)}=\tau_{n}\left((\cdot)^{-k}\right), \quad P^{(k-1)}=I\left(P^{(-k)}\right), \quad k=1,2, \ldots
$$

We sometimes write $P^{(-k)}$ and $P^{(k-1)}$ as $P_{n}^{(-k)}$ and $P_{n}^{(k-1)}$ to indicate the dependence on $n$. We have

Theorem 3 (T. Qian, 1997, [36]) Let $k$ be a positive integer. Then for any dimension number $n$, (i) $P^{(-1)}=E$; (ii) $P^{(k-1)}(x)=(-1)^{k} /(k-1) !\left(\frac{\partial}{\partial x_{0}}\right)^{k-1} E(x)$; (iii) Both $P^{(-k)}$ and $P^{(k-1)}$ are monogenic; (iv) $P_{n}^{(-k)}$ is homogeneous of degree $n+1-k$ and $P_{n}^{(k-1)}$ is homogeneous of degree $k-1$; (v) $c_{n} P_{n-1}^{(-k)}\left(x_{0}+x_{1} \mathbf{e}_{1}+\cdots+x_{n-1} \mathbf{e}_{n-1}\right)=\int_{-\infty}^{\infty} P_{n}^{(-k)}(x) d x_{n}$, where $c_{n}=$ $\int_{-\infty}^{\infty}\left(1+t^{2}\right)^{-(n+1) / 2} d t$; (vi) $P^{(-k)}=I\left(P^{(k-1)}\right)$; (vii) if $n$ is odd, then $P^{(k-1)}=\tau_{n}\left((\cdot)^{n+k+2}\right)$.

The approach of using Fourier multiplier operators is related to the Bochner type relation (for the restricted case $j=\alpha=1$ ): In the tempered distribution sense (see [49]), writing briefly ( )^ as $\mathcal{F}()$, we have

$$
\left(\frac{Q_{j}(\cdot)}{|\cdot|^{j+(n+1)-\alpha}}\right)^{\wedge}(\xi)=\gamma_{j, \alpha} \frac{Q_{j}(\xi)}{|\xi|^{j+\alpha}}, \quad j \in \mathbf{Z}^{+}, 0<\alpha<n+1
$$

where $Q_{j}$ is a polynomial, harmonic and homogeneous of degree $j$, and

$$
\gamma_{j, \alpha}=\mathbf{i}^{j} \pi^{(n+1) / 2-\alpha} \frac{\Gamma(j / 2+\alpha / 2)}{\Gamma(j / 2+(n+1) / 2-\alpha / 2)} .
$$

The relation (1) is equivalent to

$$
\int_{\mathbf{R}_{1}^{n}} \frac{Q_{j}(x)}{|x|^{j+(n+1)-\alpha}} \hat{\phi}(x) d x=\mathbf{i}^{j} \pi^{(n+1) / 2-\alpha} \frac{\Gamma(j / 2+\alpha / 2)}{\Gamma(j / 2+(n+1) / 2-\alpha / 2)} \int_{\mathbf{R}_{1}^{n}} \frac{Q_{j}(x)}{|x|^{j+\alpha}} \phi(x) d x,
$$

where $j \in \mathbf{Z}^{+}, 0<\alpha<n+1$, and $\phi$ is any test function in the Schwartz class in $\mathbf{R}_{1}^{n}$. 
The theorem asserts that if we have a Laurent series $f_{0}(z)=\sum_{k=-\infty}^{\infty} a_{k} z^{k}$, where $a_{k}^{\prime} s$ are real, then for each $n$ there is a corresponding monogenic function $\tau_{n}(f)(x)=\sum_{k=-\infty}^{\infty} a_{k} P^{(k)}$. In such way every holomorphic function on an annulus in the complex plane is associated with a monogenic function on an annulus in $\mathbf{R}_{1}^{n}$. In fact, the term $a_{k} P^{(k)}$ is identical with the projection of the sum of the monogenic functions onto the space of homogeneous monogenics of degree $|k|-n-1$ or degree $k$, depending on $k$ being negative or positive. Both series play roles as convolution kernels functions on, respectively, Lipschitz perturbations of the unit circle and the unit sphere. This formulation is shown to be crucial in the singular integral operator study in the two contexts (see next section). The assertion (vii) amounts to say that when $n$ is odd, the effects of the pointwise differential operator $\kappa_{n}^{-1} \Delta^{\frac{n-1}{2}} \tilde{f}_{0}$ and Fourier multiplier operator $\tau_{n}\left(f_{0}\right)$ are the same. Due to the result (vii) we can refer Sce's and Qian's generalizations together as the Sce-Qian generalization or the Fueter Theorem in $\mathbf{R}_{1}^{n}$.

\section{Generalizations of Fueter's Theorem of Axial Types with Monogenic factors}

The breakthrough of the Fueter type Theorems with monogenic factors is due to F. Sommen $([48])$.

Theorem 4 (F. Sommen, 1999, [48]) Let $n$ be an odd positive integer. Let $f_{0}$ be a holomorphic function in an open and connected set $\Omega$ in the upper-half complex-plane. Let $P_{k}$ be any polynomial in $\underline{x}$ of homogenicity $k$, where $k$ is a non-negative integer, and left-monogenic with respect to the homogeneous Dirac operator $\underline{D}=\sum_{l=1}^{n} \mathbf{e}_{l} \frac{\partial}{\partial x_{l}}$, ie., $\underline{D} P(\underline{x})=0$, then

$$
D\left[\Delta^{\frac{k+n-1}{2}}\left(\tilde{f}_{0}(x) P_{k}(\underline{x})\right)\right]=0 .
$$

When $k=0$ the above result reduces to Fueter's Theorem.

K.-I. Kou, T. Qian and F. Sommen extend the above result to more cases including one for $n$ being even positive integers.

Theorem 5 (K.-I. Kou, T. Qian, F. Sommen, 2002, [20]) Let $n$ be any positive integer, $f_{0}$ be an intrinsic function defined in an intrinsic domain $\Omega$ in the complex plane. Let $P_{k}$ be any function in $\underline{x}$ of homogenicity of degree $k$ and left-monogenic with respect to the homogeneous Dirac operator $\underline{D}=\sum_{l=1}^{n} \mathbf{e}_{l} \frac{\partial}{\partial x_{l}}$, then

$$
D\left[\Delta^{\frac{k+n-1}{2}}\left(\tilde{f}_{0}(x) P_{k}(\underline{x})\right)\right]=0, \quad x \in \tilde{\Omega},
$$

whenever $k+(n-1) / 2$ is a non-negative integer. If $n$ is even, then the monogenicity of the above expression also holds for $k$ being any non-negative integer and $P_{k}(\underline{x})$ a homogeneous left monogenic polynomial of degree $k$. 
In dealing with the case $n$ being even, again the Fourier multiplier method is employed. We need to first extend the relation (1) to the cases $\operatorname{Re}(\alpha)>-j, j \in \mathbf{Z}^{+}$, where $\mathbf{Z}^{+}$is the set of positive integers. In a symmetric form it was proved as

Lemma 1 ([20]) For $-j<\beta, \alpha<(n+1)+j, \alpha+\beta=n+1, j \in \mathbf{Z}^{+}$, we have

$$
\pi^{\beta / 2} \Gamma\left(\frac{j+\beta}{2}\right) \int_{\mathbf{R}_{1}^{n}} \frac{Q_{j}(x)}{|x|^{j+\beta}} \hat{\phi}(x) d x=\mathbf{i}^{j} \pi^{\alpha / 2} \Gamma\left(\frac{j+\alpha}{2}\right) \int_{\mathbf{R}_{n}^{1}} \frac{Q_{j}(x)}{|x|^{j+\alpha}} \phi(x) d x,
$$

where $\phi$ is in the Schwartz class in $\mathbf{R}_{1}^{n}$.

To prove the even dimensional case in Theorem 5, we, in particular, need the following intertwining relation which itself is based on the fundamental solution results of the operator $D \Delta^{k+(n-1) / 2}([28])$.

Lemma 2 ([20]) Let $n$ be an even positive integer. For $s=k+(n-1) / 2$, we have

$$
\left(D \Delta^{s}\right)\left(\frac{\bar{x}}{|x|^{(n+1)-2 s}} g\left(x^{-1}\right)\right)=\alpha_{n, s} \frac{\bar{x}}{|x|^{(n+1)+2 s+2}}\left(D \Delta^{s} g\right)\left(x^{-1}\right),
$$

where $\alpha_{n, s}$ is a constant depending on $n$ and $s$, and $g$ is any infinitely differentiable function in $\mathbf{R}_{1}^{n} \backslash\{0\}$.

The induced function $\tilde{f}_{0}$ from an initial complex holomorphic function is of the type $A\left(x_{0},|\underline{x}|\right)+$ $\underline{\underline{x}} B\left(x_{0},|\underline{x}|\right)$, where $A$ and $B$ satisfy the Riesz system. In general, a function defined in $\mathbf{R}_{1}^{n}$ with values in the related Clifford algebra has the form

$$
f(x)=\sum_{S} f_{S}\left(x_{0}, x_{1}, \ldots, x_{n}\right) \mathbf{e}_{S}, \quad x=x_{0}+\underline{x},
$$

where $f_{S}$ 's are scalar-valued, $S$ runs over all ordered sets $S=\left(s_{1}, \ldots, s_{j}\right)$ with $1 \leq s_{1}<\cdots<$ $s_{j} \leq n, \mathbf{e}_{S}=\mathbf{e}_{s_{1}} \cdots \mathbf{e}_{s_{j}}$, or $S=\emptyset, \mathbf{e}_{\emptyset}=1$, and $\mathbf{e}_{S}$ is called a reduced basis product with order $|S|=j$. The formal polarization of the function $f$, denoted by $\tilde{f}$, called induced function from $f$, is a function in $\mathbf{R}_{1}^{q_{1}+\cdots+q_{n}}$, defined by

$$
\tilde{f}\left(x_{0}, \underline{x}^{(1)}, \ldots, \underline{x}^{(n)}\right)=\sum_{S} f_{S}\left(x_{0}, r_{1}, \ldots, r_{n}\right) \frac{\underline{x}^{\left(s_{1}\right)}}{\left|\underline{x}^{\left(s_{1}\right)}\right|} \cdots \frac{\underline{x}^{\left(s_{j}\right)}}{\left|\underline{x}^{\left(s_{j}\right)}\right|},
$$

where $\underline{x}^{\left(s_{k}\right)} \in \mathbf{R}^{q_{k}}, r_{k}=\left|\underline{x}^{\left(s_{k}\right)}\right|, k=1, \ldots, n$. such a form is called an axial type (function) of order $m$, where $m=\max \left\{|S| \mid f_{S} \neq 0\right\}$. When $m=1$, we regard it as an axial type of order 1 ; and when $m>1$ an axial type of order $m>1$ or poly-axial type. In particular, if $m=2$ we call it biaxial type (see also [6]). If an induced function $\tilde{f}$ happens to be monogenic, then the coefficients $f_{S}$ satisfy a system of equations, usually called Vekua system, of which the simplest form, corresponding to axial type of order 1 (in other words, to para-vector-valued $\tilde{f}$ ), is the Riesz system corresponding to complex holomorphic functions $f_{0}$, or the conjugate harmonic system ([50], [19]). We also encounter functions with the form

$$
\tilde{f}\left(x_{0}, \underline{x}^{(1)}, \ldots, \underline{x}^{(n)}\right) P_{k}\left(x_{0}, \underline{x}^{(1)}, \ldots, \underline{x}^{(n)}\right),
$$


where $P_{k}$ is a monogenic function, homogeneous of degree $k$. We call such functions axial type with a monogenic factor. Accordingly, the result of Sommen and that of Kou-Qian-Sommen can be regarded as axial type order 1 generalizations of Fueter's Theorem with a monogenic factor.

The next is a similar result but for harmonic functions inspired by the computation of Sommen in [48]. We show that from harmonic functions in lower dimensions we can obtain harmonic functions in higher dimensions. Below we take the example $h(s, t)$ that is harmonic in two real variables $s$ and $t$. Suppose that we have two copies of Euclidean spaces $\mathbf{R}^{p}$ and $\mathbf{R}^{q}$ whose Clifford basis elements are, respectively, $\mathbf{e}_{1}, \ldots, \mathbf{e}_{p}$ and $\mathbf{e}_{p+1}, \ldots, \mathbf{e}_{p+q}$, where $p+q=m$, and the basis elements $\mathbf{e}_{1}, \ldots, \mathbf{e}_{m}$ satisfy the usual Clifford basis conditions for $\mathbf{R}^{m}$. Denote the variable of $\mathbf{R}^{p}$ by $\underline{x}$, and that of $\mathbf{R}^{q}$ by $\underline{y}$.

Theorem 6 (T. Qian and F. Sommen, 2003, [42]) Let $h=h(s, t)$ be harmonic in the variables $s$ and $t$ in a region in the set $\{s>0, t>0\} \subset \mathbf{R}^{2}$. Then, for any positive integer $k$,

$$
\Delta^{k}(h(|\underline{x}|,|\underline{y}|))=\sum_{l=0}^{k} \frac{k !}{l !(k-l) !} d_{p}(l) d_{q}(k-l) D_{s}(l) D_{t}(k-l) h(s, t),
$$

where the Laplacian $\Delta$ is for $p+q$ real variables:

$$
\Delta=\left(\partial_{x_{1}}^{2}+\cdots+\partial_{x_{p}}^{2}\right)+\left(\partial_{y_{1}}^{2}+\cdots+\partial_{y_{q}}^{2}\right),
$$

$s=|\underline{x}|, t=|\underline{y}|, d_{p}(l)=(p-1) \cdots(p-2 l+1), d_{p}(0)=1, D_{s}(l)=\left(\frac{1}{s} \partial_{s}\right)^{l}$. The notation $d_{q}(l)$ and $D_{t}(l)$ are defined similarly.

In particular, when $p$ and $q$ are odd, then

$$
\Delta^{\frac{p+q}{2}} h(|\underline{x}|,|\underline{y}|)=0 .
$$

It is to be noted that Fueter's and Sce's Theorems are consequences of this result. This result can be further generalized to $n$ copies of Euclidean spaces under the requirements that the space dimensions $q_{1}, \ldots, q_{n}$ are all odd numbers and $m=\sum_{l=1}^{n} q_{l}$ is even.

Technically the proof of Theorem 6 depends on expansions of powers of the operator $\frac{1}{s} \partial_{s}$.

Lemma 3 ([42]) For any $l$ numbers $c_{l}^{j}, j=1, \ldots, l, c_{l}^{l}=1$ the following four groups of conditions are equivalent.

$$
\begin{gathered}
\text { (i) } \\
D_{s}(l)=\left(\frac{1}{s} \partial_{s}\right)^{l}=c_{l}^{1} \frac{1}{s^{2 l-1}} \partial_{s}+c_{l}^{2} \frac{1}{s^{2 l-2}} \partial_{s}^{2}+\cdots+c_{l}^{l} \frac{1}{s^{l}} \partial_{s}^{l} . \\
\text { (ii) } c_{l}^{1}=(-1)^{l-1}(2 l-3) ! !, c_{l}^{j}+c_{l}^{j+1}[-(2 l-j-1)]=c_{l+1}^{j+1}, c_{l}^{l+1}=0 . \\
\text { (iii) } \\
c_{l}^{1}=(-1)^{l-1}(2 l-3) ! !, \text { and }(2 l-j) c_{l}^{j}=j c_{l+1}^{j+1} . \\
c_{l}^{j}=(-1)^{l-j} \frac{(2 l-j-1) !(2 l-2 j-1) ! !}{(j-1) !(2 l-2 j) !} .
\end{gathered}
$$

Theorem 6 implies the following extension of Fueter's Theorem. 
Theorem 7 (T. Qian and F. Sommen, [42]) Let $h(\underline{s})$ be a vector-valued function with expansion $h(\underline{s})=\sum_{r=1}^{n} h_{r}(\underline{s}) \mathbf{j}_{r}$, where $\mathbf{j}_{r}, r=1, \ldots, n$, are Clifford basis elements, and $h$ be left-monogenic with respect to the Dirac operator $\underline{\partial}_{s}=\sum_{r=1}^{n} \partial_{s_{1}} \mathbf{j}_{1}+\cdots+\partial_{s_{d}} \mathbf{j}_{n}$. Then the function

$$
\Delta^{\frac{m}{2}-1}\left(\sum_{r=1}^{n} h_{r}\left(\left|\underline{x}^{(1)}\right|, \ldots,\left|\underline{x}^{(d)}\right|\right) \frac{\underline{x}^{(r)}}{\left|\underline{x}^{(r)}\right|}\right)
$$

is left monogenic with respect to $\underline{\partial}=\sum_{r=1}^{n} \underline{\partial}_{r}$, and $\underline{\partial}_{r}=\sum_{i=1}^{q_{r}} \partial_{x_{i}^{(r)}}^{(r)}$, provided that $q_{r}$ are all odd and $m=\sum_{r=1}^{n} q_{r}$ is even.

Theorem 7 is also regarded as a axial type of order 1 generalization of Fueter's Theorem . The following result of D. Peña Peña, T. Qian and F. Sommen extends the Theorem 7 in [42] to an axial type order 1 with a monogenic factor in $\underline{x}$. Note that the proof is based on further analysis of powers of the operator $\frac{1}{s} \partial_{s}$.

Theorem 8 (D. Peña Peña, T. Qian, F. Sommen, 2006, [29]) Let

$$
g\left(x_{0}, r_{1}, \ldots, r_{n}\right)=\left(g_{0}\left(x_{0}, r_{1}, \ldots, r_{n}\right), g_{1}\left(x_{0}, r_{1}, \ldots, r_{n}\right), \ldots, g_{d}\left(x_{0}, r_{1}, \ldots, r_{n}\right)\right),
$$

where the scalar-valued functions $g_{0}, g_{1}, \ldots, g_{n}$ satisfy the conditions for a conjugate harmonic system ([50]). Let the dimensions of the Euclidean spaces be $q_{1}, \ldots, q_{n}$, and with the vectors $\underline{x}^{(1)}, \ldots, \underline{x}^{(n)}$, respectively, and $m=\sum_{r=1}^{n} q_{r}$. If all $q_{k}$ are odd and $m-n$ is even, then

$$
\Delta^{k+(m-n) / 2}\left[\left(g_{0}\left(x_{0}, r_{1}, \ldots, r_{n}\right)+\sum_{j=1}^{d} g_{j}\left(x_{0}, r_{1}, \ldots, r_{d}\right) \frac{\underline{x}^{(j)}}{\left|\underline{x}^{(j)}\right|}\right) P_{k}(\underline{x})\right]
$$

is left monogenic with respect to $\underline{x}=\left(\underline{x}^{(1)}, \ldots, \underline{x}^{(n)}\right)$, where $r_{j}=\left|\underline{x}^{(j)}\right|, P_{k}\left(\underline{x}_{)}=\prod_{j=1}^{n} P_{k_{j}}^{(j)}\left(\underline{x}^{(j)}\right), k=\right.$ $\sum_{j=1}^{k} k_{j}$, where each $P_{k_{j}}^{(j)}$ is a monogenic function homogeneous of degree $k_{j}$ with values in the related Clifford algebra.

In [42] and [29] the authors deal with monogenic para-vector-valued initial functions that correspond to sets of functions satisfying the Riesz system or, in other words, conjugate harmonic system ([50], [19]). According to the above defined terminology, they correspond to axial type of order 1. In the papers [30] and [31] the authors present how it is possible to start from a monogenic function in a certain Clifford algebra, not necessarily being of the para-vectorvalued type, to generate monogenic functions in other Clifford algebras of higher dimensions corresponding to axial types of order $m>1$.

Next, in [32], it is proved that in the preceding cases, if the homogeneous monogenic factor $P_{k}(\underline{x})$ in $\mathbf{R}^{m}$ is replaced by a homogeneous monogenic polynomial $P_{k}\left(x_{0}, x\right)$ in $\mathbf{R}_{1}^{m}$, then the corresponding Fueter type theorem still holds. Additionally, Theorem 2 of the paper shows that Fueter's Theorem can be generalized to the initial functions $f_{0}$ satisfying $\bar{\partial}_{z} \Delta^{k} f(z)=0$ instead of being holomorphic.

The above mentioned cover most general Fuether type theorems so far if the involved power of the Laplacian is a point-wise differential operator. The technical proofs invoke properties of 
powers of the operator $\frac{1}{s} \partial_{s}$. We note that in the lately summarized papers, approximately since [42], the counterpart results corresponding to fractional powers of the Laplacian, in fact, remain unproved.

\section{Applications of Fueter's Theorem and its Sce-Qian General- ization}

\subsection{Applications to $H^{\infty}$-functional calculi of Dirac Operators}

It is a fact that before [37] there had been no real applications of Fueter's Theorem apart from the Fueter type relations between certain kernels indicated in the end of $\S 1$. The first real application of Fueter's Theorem would be one given by Qian in [37] to prove boundedness of singular integral operators that involve infinitely many concrete examples. In [37] the theory of bounded holomorphic functional calculus (Cauchy-Dunford type) of the Dirac operator on Lipschitz perturbations of the three dimensional unit sphere centered at the origin in the quaternionic space is established. Below we give a brief description of the theory. This theory was initialized by Alan McIntosh and developed in a series of papers by himself and his fellows.

In the one-dimensional case the Dirac operator on the line is $\frac{1}{i} \frac{d}{d x}$ whose spectrum set is identical with the unbounded set $(-\infty, 0) \cup(0, \infty)$ [26]. The classical Cauchy-Dunford bounded holomorphic functional calculus theory cannot be applied in this case. It is found that the functional calculus theory is available not only on the line, but also on Lipschitz graphs as Lipschitz perturbations of the real line. The theory shows that the operators in the Cauchy-Dunford type functional calculus, being an operator algebra, has other two equivalent forms, viz., the bounded holomorphic $\left(H^{\infty}\right)$ Fourier multipliers under the initialization of Fourier transformation on curves by Coifman and Meyer, and singular integral operators with holomorphic kernels ([25], [26]). It is noted that the success of the formulation of such functional calculus is guaranteed by the celebrated CMcM Theorem in 1986 solving the long standing Calderón conjecture on $L^{2}$ boundedness of the Cauchy singular integral operator on Lipschits curves ([8]). The Calderón conjecture is closely related to boundary value problems and Hardy spaces in Lipschitz domains ([52], [22]). The involved techniques were further developed to establish the so called $T(b)$ Theorem. The boundedness of the functional calculus was proved by using eventually the same techniques as those as used to prove the CMcM Theorem. Following the same idea McIntosh et al established an analogous theory on Lipschitz surfaces in the Clifford algebra setting ([23], [24], [17]), where the linear differential operator $\frac{1}{i} \frac{d}{d x}$ on the real-line case is replaced by the Dirac operator $D$ or $\underline{D}$. Qian and his collaborators were responsible for establishment of the theory on Lipschitz perturbations of the unit circle and those of the unit spheres in $\mathbf{R}^{n}$ and $\mathbf{R}_{1}^{n}$ with respect to the spherical Dirac operator ([35], [18], [37], [38]). Cowling and Qian published a counterpart result on the $n$-complex unit sphere with respect to Euler's operator $z \frac{z}{d z}([7])$. Each of those functional calculi consists of singular integrals of holomorphic or monogenic kernels that have similar properties like the Hilbert transformation in the individual contexts ([43], [1], [2]). 
Establishing a singular integrals theory for spheres is technically different from establishing the same theory on the unit circle. For the latter a periodization technique in relation to Poisson summation formula can be adopted and the theory for the circle and its Lipschitz perturbations follows from those of the non-periodic case on the real line ([14], [35], [18], [34]). In higher dimensions similarly an analysis theory on the n-torus may be obtained from the corresponding one on the cubes. The theory for spheres, however, is not a periodization of the theory on the plane, and there is no counterpart Poisson summation formula in relation to spheres. In gaining a counterpart singular integrals theory for spheres in higher dimensions Fueter's Theorem, its Sce-Qian generalizations and the related estimates are found to be crucially necessary. It is also the unique methodology so far. As a matter of fact, establishing the Cauchy-Dunford functional calculi of the spherical Dirac operators motivated Qian to generalize Fueter's and Sce's theorems.

Below we have a closer look into the formulation of the functional calcului on Lipschitz perturbations of the spheres ([38]). Let $S_{\omega}^{c} \subset \mathbf{C}$ be the open double sector with opening angle $2 \omega, 0<\omega<\pi / 2$, symmetric with respect to the real- and the imaginary-axes, and containing the open set $(-\infty, 0) \cup(0, \infty)$. Consider the function class $H^{\infty}\left(S_{\omega}^{c}\right)$ consisting of bounded holomorphic functions intrinsically defined on $S_{\omega}^{c}$. We can show, as in [23], that each such $b$ in $H^{\infty}\left(S_{\omega}^{c}\right)$ is associated with a singular integral kernel $\phi$ on a certain neighborhood of sphere excluding a point. The kernel is monogenic in a corresponding open set in $\mathbf{R}_{1}^{n}$ with singularity $-n$ at the origin which is the same as the Hilbert transformation on the sphere ([43]). The set of such monogenic kernels $\phi$ can be characterized in terms of their singularity degrees so to have a one to one correspondence with the set of the functions $b$ in $H^{\infty}\left(S_{\omega}^{c}\right)$. In [38] we show that $C_{\phi}=M_{b}=b\left(D_{\Sigma}\right)$, where $C_{\phi}$ denotes the singular integral operator on the sphere induced by $\phi$; $M_{b}$ stands for the Fourier multiplier operator on the sphere induced by $b$; and $b\left(D_{\Sigma}\right)$ stands for the Cauchy-Dunford functional calculus expressed as contour integral around the spectrum set of the Dirac operator $D_{\Sigma}$ on the Lipschitz surface $\Sigma$. Such defined operators form an operator algebra. On the sphere the Plancherel Theorem is valid that implies the $L^{2}$-boundedness of the operators. Since the surface measure of the sphere satisfies the doubling measure condition the $L^{2}$-boundedness implies the weak- $L^{1}$ boundedness. By the routine method the BMO boundedness can also be obtained. The operator interpolation method then can be used to obtain the $L^{p}$-boundedness for $1<p<\infty$. On the Lipschitz perturbations the formulation of the three equivalent forms of the $H^{\infty}$-functional calculus is still valid. To show the boundedness for the operators on the Lipschitz surfaces, however, is much more difficult. That is essentially due to the fact that the Plancherel theorem does not hold on Lipschitz surfaces. What we use are techniques generalized from the proofs of the CMcM Theorem. In order to do so we heavily rely on the right estimates of the kernels that invoke Fuether's Theorem and its higher dimensional generalizations, viz., the Sce-Qian Theorem.

In $\mathbf{R}_{1}^{n}$ we shall be working on heart-shaped regions and their complements

$$
H_{\omega, \pm}=\left\{x \in \mathbf{R}_{1}^{n} \mid \frac{ \pm \log |x|}{\arg \left\langle\mathbf{e}_{\mathbf{0}}, \mathbf{x}\right\rangle}<\tan \omega\right\}
$$

and

$$
H_{\omega}=H_{\omega,+} \cap H_{\omega,-} .
$$

That is,

$$
H_{\omega}=\left\{x \in \mathbf{R}_{1}^{n} \mid \frac{|\log | x||}{\arg \left\langle\mathbf{e}_{\mathbf{0}}, \mathbf{x}\right\rangle}<\tan \omega\right\}
$$


We also use the following function class for kernels.

$K\left(H_{\omega, \pm}\right)=\left\{\phi: H_{\omega, \pm} \rightarrow C^{(n)}: \phi\right.$ is monogenic and satisfies $\left.|\phi(x)| \leq C_{\mu} /|1-x|^{n}, x \in H_{\mu, \pm}, 0<\mu<\omega\right\}$, and

$$
K\left(H_{\omega}\right)=\left[\left\{\phi: H_{\omega} \rightarrow C^{(n)} \mid \phi=\phi_{+}+\phi_{-}, \phi_{ \pm} \in K\left(H_{\omega, \pm}\right)\right\} .\right.
$$

Denote by $S_{\omega, \pm}^{c}$, respectively, the open half-sectors in the complex plane that have opening angle $2 \omega$, contain the corresponding open half-real axes and are symmetric to them. By $H^{\infty}\left(S_{\omega, \pm}^{c}\right)$ we denote, respectively, the sets of bounded and holomorphic functions on $S_{\omega, \pm}^{c}$. We have

Theorem 9 (T. Qian, 2001, [38]) If $b \in H^{\infty}\left(S_{\omega, \pm}^{c}\right)$ and $\phi(x)=\sum_{k= \pm 1}^{ \pm \infty} b(k) P^{(k)}(x)$, then $\phi \in$ $K\left(H_{\omega, \pm}\right)$.

Theorem 10 (Qian, 2001, [38]) If $b \in H^{\infty}\left(S_{\omega}^{c}\right)$ and $\tan \omega$ is larger than the Lipschitz constant of a Lipschitz perturbation of the unit sphere, then $C_{\phi}=M_{b}=b\left(D_{\Sigma}\right)$, where $C_{\phi}$ is the singular integral operator on the sphere induced by $\phi, M_{b}$ stands for the Fourier multiplier operator on the sphere induced by $b$, and $b\left(D_{\Sigma}\right)$ stands for the Cauchy-Dunford functional calculus for $b$ defined through a contour integral around the spectrum set of the spherical Dirac operator $D_{\Sigma}$. Such defined operators form a bounded operator algebra from $L^{p}$ to $L^{p}$ of the Lipschitz surface for $1<p<\infty$, and bounded from $L^{1}$ to weak- $L^{1}$.

We note that $H^{\infty}$-functional calculi of the Dirac operators on the Lipschitz curves and surfaces is one of the two main themes extending the CMcM Theorem. In each of the studied contexts we extend the singular Cauchy operator to its largest possible extent in which the corresponding kernels are monogenic, and the associated operators are bounded and form an operator algebra. The results are in the line of the so called A. Calderón program in relation to pseudo-differential operators that can be used to solve certain types of partial differential equations. The other extension of the CMcM Theorem was proposed and studied mainly by $\mathrm{G}$. David and S. Semmes. They extend Lipschitz curves and surfaces to regular curves and surfaces. The kernels they concern, however, are only of the Cauchy type, including odd rational functions ([9], [10], [45], [46], [47]).

Another direction of singular integral study on curves and surfaces, yet different from the above mentioned two in its nature, is based on conformal mappings. Conformal mappings introduce conformal weights (Jacobi of conformal change of variable) on curves and surfaces ([40], [39]). As result, the underlying measure is not the natural surface area. From the analysis point of view this formation does not add essentially new knowledge to singular integral theory. 


\subsection{Applications to Computations of kernels and Generating Functions of the Clifford-Hermite and Clifford Gegenbauer Polynomials}

Almost all kernels in higher dimensional spaces are deducible by employing Fueter type theorems: Higher dimensional Newton potentials and Poisson kernels are easily deducible from the corresponding objects in the $\mathbf{R}^{2}$ space. Even higher dimensional heat kernels are deducible in such way from the corresponding objects in the lower dimensions, although heat kernels do not fit into our theory (see [42]).

In papers [33], [11] and [12] generalizations of Fueter's theorem are used to find explicit formulae of the generating functions of the Clifford-Hermite and Clifford-Gegenbauer polynomials.

\section{Inverse of the Fueter Mapping}

The problem of the inversion of the Fueter mapping has been addressed in recent time, see $[3,4,5,6]$. In [4] the authors solve the problem of finding a Fueter primitive of an axially monogenic function. Specifically, assume that $x=x_{0}+r \underline{\omega} \in \mathbf{R}^{n+1}, n$ odd, and $F(x)=$ $A\left(x_{0}, r\right)+\underline{\omega} B\left(x_{0}, r\right)$ is a given axially monogenic function. The problem consists in finding out a function $f(x)=\alpha\left(x_{0}, r\right)+\underline{\omega} \beta\left(x_{0}, r\right)$, where $\alpha, \beta$ satisfy the Cauchy-Riemann system, such that $\Delta^{(n-1) / 2} f=F$. In [4] the authors provide a Fueter primitive of an axially type monogenic function of order $k$. The same problem is solved in [3] with a different method. In [6] a Fueter primitive of a biaxial monogenic function is provided. Finally, in [21] it is shown that the basic result in [4] in finding a Fueter's primitive can also be extended to $\mathbf{R}_{1}^{n}$ for even $n$ 's.

\section{Conclusion}

Generalizations of Fueter's Theorem have been undergoing great development since 1935, and, till now, finding themselves large spaces to be further developed. The generalizations were motivated by applications in other branches of mathematics including functional calculus and operator algebra. It is anticipated that the current studies on inverse Fueter theorems would explore more involvements in mathematics and, in particular, applications to analysis of multivariable functions.

\section{References}

[1] A. Axelsson, K. I. Kou and T. Qian, Hilbert transforms and the Cauchy integral in Euclidean space, Studia Mathematica, 2009, 193(2), 161-187.

[2] S. Bell, The Cauchy transform, potential theory and conformal mappings, 1992. 
[3] F. Colombo, D. Peña Peña, I. Sabadini, and F. Sommen, A new integral formula for the inverse Fueter mapping theorem, preprint 2013.

[4] F. Colombo, I. Sabadini and F. Sommen, The inverse Fueter mapping theorem. Commun. Pure Appl. Anal. 10 (2011), no. 4, 1165-1181.

[5] F. Colombo, I. Sabadini and F. Sommen, The inverse Fueter mapping theorem in integral form using spherical monogenics. Israel Journal of Mathematics, 194 (2013), 485-505.

[6] F. Colombo, I. Sabadini and F. Sommen, The Fueter primitive of biaxially monogenic functions, to appear in Comm. Pure Appl. Anal.

[7] M. Cowling, T. Qian, A class of singular integrals on the $n$-complex unit sphere, Science in China (Series A), December 1999, Vol. 42 No. 12, 1233-1245.

[8] R. Coifman, A. McIntosh and Y. Meyer, L'intégrale de Cauchy définit un opérateur borné sur L2 pour les courbes lipschitziennes, Ann. of Math. 116 (1982), 361-387.

[9] G. David, Opérateurs intégraux singuliers sur certains courbes du plan complexe, Ann. Sci. Ećole Norm. Sup. 17 (1984), 157-189.

[10] G. David, Wavelets and Singular Integrals on Curves and Surfaces, Lecture Notes in Mathematics, Vol. 1465, Springer-Verlag, Berlin-New York.

[11] H. De Bie, D. Peña Peña and F. Sommen, The generating function of the CliffordGegenbauer polynomials, AIP Conf. Proc. 1479, 321-324 (2012).

[12] H. De Bie, D. Peña Peña and F. Sommen, Generating functions of orthogonal polynomials in higher dimensions, submitted for publication.

[13] R. Delanghe, F. Sommen and V. Soucek, Clifford Algebra and Spinor-Valued Functions, Vol. 53, Kluwer Academic Publishers, Dorderecht, Boston, London, 1992.

[14] R.E. Edwards and G.I. Gaudry, Littlewood-Paley and Multiplier Theory, SpringerVerlag, Berlin, Heidleberg, New York, 1977.

[15] R. Fueter,Die Funktionentheorie der Differentialgleichungen $A M=0$ und AAit $=0$ mit vier reellen Variablen, Comment. Math. Helv. 7 (1935), 307-330.

[16] J.B. Garnett, Bounded Analytic Functions, Academic Press: 1987.

[17] G. Gaudry, R.-L. Long, and T. Qian, A martingale proof of L2-boundedness of Cliffordvalued singular integrals, Ann. Math. Pura Appl. 165 (1993), 369-394.

[18] G. Gaudry, T. Qian, S.-L. Wang, Boundedness of singular integrals with holomorphic kernels on star-shaped closed Lipschitz curves, Colloq. Math, Vol.LXX (1996),133-150.

[19] J. Gilbert and M. Murray, Clifford Algebra and Dirac Operator in Harmonic Analysis, Cambrage University Press: Cambridge, MA 1991.

[20] K.-I. Kou, T. Qian, F. Sommen, Generalizations of Fueter's Theorem, Methods and Applications of Analysis, Vol. 9, No. 2, pp. 273-290, June 2002. 
[21] K.-I. Kou, T. Qian, I. Sabadini, The inversion theorem of Fueter's Theorem in $\mathbf{R}_{1}^{n}$ for even n's, preprint.

[22] , C. E. Kenig, Harmonic analysis techniques for second order elliptic boundary value problems, CBMS, Regional Conference Series in Mathematics, Nmber 83, 1991.

[23] C. Li, A. McIntosh, and T. Qian, Clifford algebras, Fourier transforms, and singular convolution operators on Lipschitz surfaces, Rev. Mat. Iberoamericana 10 (1994), 665721.

[24] C. Li, A. McIntosh, and S. Semmes, Convolution singular integrals on Lipschitz surfaces, J. Amer. Math. Soc. 5 (1992), 455-481.

[25] A. McIntosh and T. Qian, Convolution singular integral operators on Lipschitz curves, Proc. of the Special Year on Harmonic Analysis at Nankai Inst. of Math., Tianjin, China, Lecture Notes in Mathematics, Vol. 1494, pp. 142-162.

[26] A. McIntosh and T. Qian, Lp Fourier multipliers on Lipschitz curves, Trans. Amer. Math. Soc. 333 (1992), 157-176.

[27] Y. Meyer and R. Coifman, Wavelets, Calderón-Zygmund and Multilinear Operators, Cambridge studies in advance mathematics, 48, (English Version) Cambridge University Press, 1997.

[28] J. Peetre and T. Qian, Möbius covariance of iterated Dirac operators, J. Austral. Math. Soc., Series A, 56 (1994), PP. 665-721.

[29] D. Peña Peña, T. Qian, F. Sommen, An alternative proof of Fueter's theorem, Complex Variables and Elliptic Equations, 51 (2006), no. 8-11, 913-922.

[30] D. Peña Peña, F. Sommen, A Generalization of Fueters Theorem, Results in Mathematics, 49 (2006), no. 3-4, 301-311.

[31] D. Peña Peña, F. Sommen, A note on the Fueter theorem, Advances in Applied Clifford Algebras, 20 (2010), no. 2, 379-391.

[32] D. Peña Peña, F. Sommen, Fueter's theorem: the saga continues, Journal of Mathematical Analysis and Applications, 365 (2010), no. 1, 29-35.

[33] D. Peña Peña, F. Sommen, Monogenic Gaussian distribution in closed form and the Gaussian fundamental solution, Complex Variables and Elliptic Equations, 54 (2009), no. $5,429-440$.

[34] T. Qian, A holomorphic extension result, Complex Variables, vol 32(1)(1997), 59-77.

[35] T. Qian, Singular integrals with holomorphic kernels and Fourier multipliers on starshape Lipschitz curves, Studia Mathematica, 123(3) (1997),195-216.

[36] T. Qian, Generalization of Fueter's result to $R^{n+1}$, Rend. Mat. Acc. Lincei (9) 8 (1997), 111-117.

[37] T. Qian, Singular integrals on star-shaped Lipschitz surfaces in the quaternionic space, Math. Ann. 310(1998), 601-630. 
[38] T. Qian, Fourier Analysis on starlike Lipschitz surfaces, Journal of Functional Analysis 183 (2001), 370-412.

[39] T. Qian, X.H. Ji J. Ryan, Fourier theory under M?bius transformations, Clifford Algebras and Their Applications in Mathematical Physics, Volume2, edited by John Ryan and Wolfgang Spr?ssig, Birkh?user,Boston-Basel-Berlin (April 2000), 51-80.

[40] T. Qian and J. Ryan, Conformal transformations and Hardy spaces arising in Clifford analysis, J. Operator Theory 35 (1996), 349-372.

[41] T. Qian, X.H, Ji and J. Ryan, Fourier theory under Möbius transformations, Clifford Algebras and Their Applications in Mathematical Physics, Vol.2, Edited by J. Ryan and W. Sproessig, Birkhäuser, Boston-Basel-Berlin (April, 2000), 51-80.

[42] T. Qian and F. Sommen, Deriving harmonic functions in higher dimensional spaces, Zeitschrift fr Analysis and ihre Anwendungen (Mathematical Methods in the Applied Sciences), Vol 22 (2003)2, 275-288.

[43] by T. Qian and Y. Yang, Hilbert Transforms on the Sphere With the Clifford Algebra Setting, Journal of Fourier Analysis and Applications, (2009) 15: 753-774.

[44] R. F. Rinehard, Elements of a theory of intrinsic functions on algebras, Duke Math. J., 32, 1965, 1-19.

[45] S. Semmes, A criterion for the boundedness of singular integrals on hypersurfaces, Trans. Amer. Math. Soc. 311 (1989), 501-513.

[46] S. Semmes, Analysis vs. geometry on a class of rectifiable hypersurfaces in Rn, Indiana Univ. Math. J. 39 (1990), 1005-1035.

[47] S. Semmes, Chord-arc surfaces with small constant, $l^{*}$, Adv. Math. 85 (1991), 198-223.

[48] F. Sommen, On a generalization of Fueter's theorem, Zeitschrift für Analysis und ihre Anwendungen, Journal for Analysis and its Applications, Vol. 19 (2000), No. 4, pp. 899-902.

[49] E.M. Stein, Singular Integrals and Differentiability Properties of Functions, Princeton University, 1970.

[50] E.M. Stein and G. Weiss, Introduction to Fourier Analysis in Euclidean Spaces, Princeton University Press, 1971.

[51] A. Sudbery, Quaternionic analysis, Math. Proc. Camb. Phil. Soc., 85, 1979, 199-225.

[52] G. Verchota, Layer potentials and regularity for the Dirichlet problem for Laplace's equation, J. of Funct. Anal. 59 (1984), 572-611.hh 